\title{
The Impact of Community Structure of Social Contact Network on Epidemic Outbreak and Effectiveness of Non-pharmaceutical Interventions
}

\author{
Youzhong Wang ${ }^{1}$, Daniel Zeng ${ }^{1,2}$, Zhidong Cao ${ }^{1}$, Yong $\mathrm{Wang}^{3}$, \\ Hongbin Song ${ }^{3}$, and Xiaolong Zheng ${ }^{1}$ \\ ${ }^{1}$ The Key Laboratory of Complex Systems and Intelligence Science, \\ Institute of Automation, Chinese Academy of Sciences, Beijing, China \\ ${ }^{2}$ MIS Department, The University of Arizona, Tucson, Arizona, U.S.A. \\ ${ }^{3}$ Institute of Disease Control and Prevention, \\ Academy of Military Medical Sciences, Beijing, China \\ \{youzhong.wang, dajun.zeng, zhidong.cao\} @ia.ac.cn, \\ ywang7508@gmail.com, hongbinsong@263.com, \\ xiaolong.zheng@ia.ac.cn
}

\begin{abstract}
The topology structure of social contacts network has a big impact on dynamic patterns of epidemic spreading and effectiveness of nonpharmaceutical interventions. Corresponding to individuals' behavioral or functional units, people are commonly organized in small communities, meaning that most of social contacts networks tend to display community structure property. Through empirical investigation and Monte-Carlo simulation on a big H1N1 outbreak in a Chinese university campus, this paper explores the impact of community structure property of social contacts network on epidemic spreading and effectiveness of interventions. A stochastic model based on social contacts networks among students is constructed to simulate this outbreak, revealing that epidemic outbreaks commonly occur in local community. Moreover, effectiveness of three quarantine-based interventions is quantitatively studied by our proposed model, finding that community structure of social networks determines the effects these measures.
\end{abstract}

Keywords: community structure, social contact network, epidemic outbreak, non-pharmaceutical Interventions, H1N1.

\section{Introduction}

For many kinds of communicable diseases, contagion is spread among the population mainly through close contact such as talking and physical touch. As intensity and frequency of close contact are determined primarily by social relationships between individuals, studying patient social networks has obvious advantage to understand which reasons determined the epidemic outbreak and trend.

Over the last few years, there have been an increasing number of efforts to build variety of theoretical models that couple the classical compartmental models such as 
susceptible-infectious-recovery (SIR) model with complex social network methods. These combined models borrow the conception from compartmental models that individuals are divided into different compartments according to their states and their states are transformed with the time [1]. On the other hand, these methods overcome the limitation that assumes populations are homogeneously mixed in standard compartmental models. Recent advances in social network study provide useful tools to simulate and analyze the epidemic dynamics among the real population in which contacts between individuals are not simply selected randomly but possessing many of complex properties [2-4].

One of important properties that possessed by many real social contacts network is community structure. Community structure, also known as hierarchical organization or modularity, refers to the fact that a network can be divided into sub-groups. Nodes within these sub-groups are densely connected while the connection between subgroups is much sparser [5]. In real world, people are commonly organized in many of small communities corresponding to their behavioral or functional units such as residence district, work place, and university campus. It is found that the close and frequent contacts among population in these social groups make these local areas be highest-risk environments for outbreaks of infectious diseases, especially for respiratory infectious diseases such as $\mathrm{H} 1 \mathrm{~N} 1$ virus $[6,7]$. How to protect people from being infected in such communities has been a public health priority to many countries.

On the other hand, the community structure of social contact networks determines the effectiveness of non-pharmaceutical intervention methods to slow down infection spreading in local communities. Non-pharmaceutical interventions have the added benefit of lessening the worry that pharmaceutical interventions may induce viral drug resistance. As such, from the very beginning of the 2009 H1N1 pandemic, many countries, including China, had adopted control strategies such as quarantining travelers from foreign countries, closing the schools, among others. These control strategies gain great benefits to cut down many of social contacts among population in communities and thus prevent the epidemic outbreak. In effect, this strategy, i.e., quarantining close contacts of the infected persons, was proved to be effective against SARS infections in 2003 [8,9]. Obviously, it would cost more when we take the strategies earlier or more stringent. A quantitative evaluation of the effectiveness of different control measures is essential for us to choose when and how to conduct intervention methods. As most of contacts are within local social groups, it is essential to study the impact of community structure on the effectiveness of control strategies.

In this paper, we conduct a case study of a large 2009 pandemic influenza A (H1N1) outbreak to characterize transmission patterns of $\mathrm{H} 1 \mathrm{~N} 1$ virus in a school campus that possesses the property of community structure. According to the social contact information from a detailed epidemiologic investigation of the outbreak, we construct a hierarchical social network among students in one apartment building where a severe H1N1 outbreak occurred. A compartmental based stochastic model is proposed to simulate the spreading process of the epidemic in the network, finding community outbreaks within small social groups. Moreover, we compare the effect of three quarantine-based non-pharmaceutical interventions (including dormitory building quarantine only, plus between-room visits prohibition, and plus relocation of all people at risk to a treatment center) on transmission, aiming to find out the most efficient control measures with acceptable cost. In practical, it needs to respond to the 
infectious outbreak timely, we compare the strategies to advise how to control the spreading in an emergency.

\section{Related Work}

The past several years have seen dramatic advances in exploring the transmission pattern of infectious disease and evaluating public health response and control strategies [10]. From a methodological standpoint, these studies typically employ either case-study or simulation methods. We argue that these two types of methods can complement each other very well. Studying real-world outbreaks through epidemiological and case studies can reveal critical insights to help understand the dynamics of disease transmission and identify risk factors [11-14]. These insights can also serve as the base for the follow-up analytical modeling and computational simulation work and facilitate an understanding of the impact of various public health responses [15, 16]. However, using case studies and real-world observations alone cannot guide the selection of the optimal control or response strategies because of lack of support for comparing various alternative measures. Simulations, on the other hand, offer a complementary framework enabling us to quantitative study the impact of various strategies [17]. In particular, detailed cost and benefit analyses are possible through simulation. [18, 19].

A commonly used framework for simulation of epidemic spreading is social network. After a great development in last two decades, various kinds of complex properties are found in social network structure [20]. These system properties, which emerge from individuals' behaviors and interactions, have a big impact of epidemic transmission among persons in the network. For example, recent study [21] shows that infection would tend to spread over and stay at a steady state in a heterogeneous networks (i.e., a network in which a small fraction of individuals possess most of contacts and many of other individuals only have a few contacts, this property is present in many real networks such as [22, 23]).

It has been shown that many real social networks are hierarchical organized by linking many of small communities [5, 24]. This network property is commonly called as community structure, which greatly influences the dynamic effects on real networks $[25,26]$. For infectious disease transmission, the community structure property of social contacts network would arouse community outbreaks within small social groups, which have attracted public attention for intervention methods design in order to control epidemic spreading $[6,7]$.

\section{Data and Methodology}

\subsection{Data}

From August 28, through September 17, 2009, a big H1N1 outbreak occurred on a campus of a Chinese university near Beijing. 206 students are infected during this outbreak in total, and about a half (105) of them are living in a six-storied apartment building. In this paper, we are mainly focus on H1N1 virus spreading in this one 
building, as it was the main infection hotspot and a center of student interactions during the outbreak.

Students living in this building belonged to the same academic department, and were divided into 14 classes. The students of the same class were assigned to adjacent rooms and each room typically housed six students. Therefore, students in this dormitory are organized hierarchically from a single dormitory room to a class to the whole building. To further understand the social contacts between students, we conduct a preliminary epidemiological investigation of these confirmed cases to collect their close contacts before onset of the illness. In this investigation, 30 respondents recalled their close contact history, involving in total 45 virus carriers. We list the relationship between these respondents and their infected contacts in Table 1. Before the building was isolated, 9 respondents were mainly infected by outsiders. After the building was isolated, 21 respondents were mainly infected by roommates and in a lesser degree by classmates in other rooms. Moreover, we investigate all infected cases about their time delay from infected to be hospitalized, finding that it took about 1.5 days on average.

In order to control this outbreak, the administration of the school took several emerging interventions, including 1) isolating the apartment building to stop the interbuilding transmissions on August $31 ; 2$ ) prohibiting students in the apartment building to contact other students living in different dormitory rooms on September 3;3) quarantining all probable close contacts when a infected student is sent to the hospital in the entire outbreak.

Table 1. The relationship between the respondents and their contacts to H1N1 virus carriers

\begin{tabular}{lcccccc}
\hline & Respondents & contacts & Roommates & Classmates & $\begin{array}{c}\text { Different } \\
\text { classes }\end{array}$ & $\begin{array}{c}\text { Different } \\
\text { buildings }\end{array}$ \\
\hline $\begin{array}{l}\text { Whole period* } \\
\begin{array}{l}\text { Before isolate } \\
\text { building }\end{array}\end{array}$ & 30 & 45 & 16 & 11 & 2 & 16 \\
$\begin{array}{l}\text { After isolate } \\
\text { building }\end{array}$ & 9 & 13 & 0 & 0 & 0 & 13 \\
\hline
\end{tabular}

\subsection{Method}

As shown in Table 1, social contacts among the students in the apartment building show a community structure property, as a student has a biggest probability to be infected by his (her) roommates and has a moderate probability to be infected by his (her) classmates in different rooms and has a smallest probability to be infected by students in different classes. In order to model social contacts among students in the building, we construct a complex network by representing students as nodes and social relationships among them as links. Due to the community structure property of social contacts among students in the apartment, we allocate to each student different probabilities that contacting to his roommate, neighbors in the same classes, and others in the same building, respectively. The probabilities are preset based on the epidemiologic investigation: we assume that the contact histories of the 30 respondents in Table 1 can reflect the behaviors of other students. As the persons living in 
the same room should contact with each other determinately in each day, we obtain the average number of one's contactors who are roommates, classmates but living in other rooms, others living in the same building is $p_{1}=5, p_{2}=3.5$ and $p_{3}=0.5$, respectively. The network model is shown as Fig. 1.

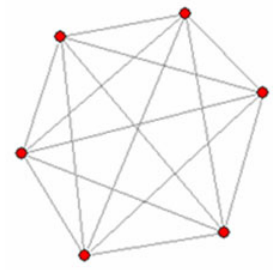

(a)

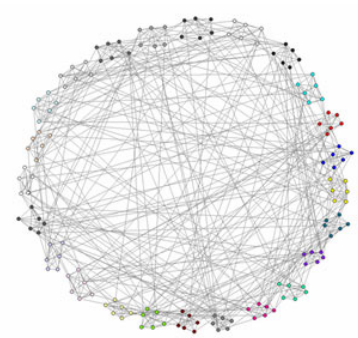

(b)

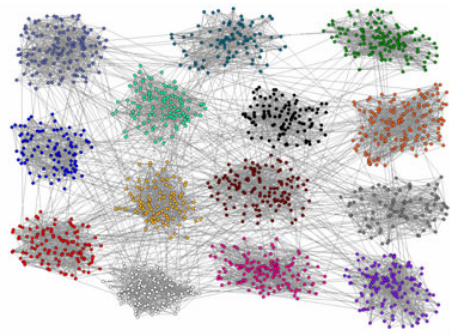

(c)

Fig. 1. (a) The social contacts network in one room. All students living in one room are fully connected. (b) The social contacts network in one class. Many edges exist in each room, and fewer edges between rooms. (c) The social contacts network in the building. The students are divided into different classes; many edges exist in each class, and fewer edges between classes.

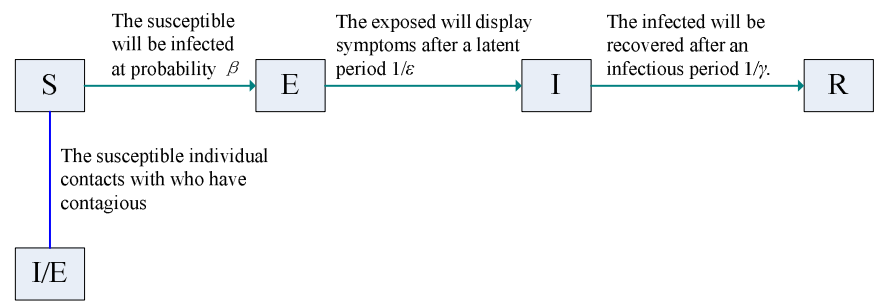

Fig. 2. The state change process of epidemic spreading in SEIR model

A stochastic SEIR model was applied to simulate the disease spreading process on the network. In the SEIR model, people are classified into four classes: Susceptible, Exposed (Incubation), Infected, and Recovered [27]. The state change process of epidemic spreading in SEIR model is shown in Fig. 2 . We set the latent period $1 / \varepsilon=2$ days according to some empirical studies of H1N1 transmission [28, 29] and theinfectious period as $1 / \gamma=1.5$ days based on our epidemiologic investigation. The spreading rate $\beta$ was set to 0.18 , which leads that the coefficient of determination, a measurement of how well the real data can be explained by the model, between the daily counts of new infections in the real outbreak and simulation results is maximum.

Following the control strategies the school administrator taken during the outbreak, we divide the outbreak into three periods: 1) From August 28 to August 31, the apartment building was not isolated, and students in the building may be infected by infectors outside the building with a probability $\lambda=0.004 ; 2$ ) From September 1 to September 3, infection can only be transmitted within the apartment building; 3) After September 3, the contacts between two students in different rooms are cut off with probability $\eta=1$, meaning that infection would not be spread inter-rooms. 
During the outbreak, the university administration investigated the infected to identify their close contacts for quarantining. In practice, however, identify all contacts successfully is difficult as it is very time-consuming to collect contacts histories of infected students. As a proxy, the roommates of a patient should be viewed as automatic close contacts and considered as candidates to be quarantined. In our simulation, we assume that all roommates of an infected patient would be quarantined when the infected patient is hospitalized, and preset the probability of identifying and quarantining a neighbor exposed to an infected is $\theta=0.8$ according to the investigation taken by the administration. Related parameters are listed in Table 2.

Using our network-based model, we have studied the impact of three different control measures on $\mathrm{H} 1 \mathrm{~N} 1$ spread. We adjust the date to implement these measures in the simulation on one hand and the parameters $\lambda, \eta, \theta$ on the other hand, in order to quantitatively examine the impact of timeliness and seriousness of control measures on control effects. The outcomes of control measures are measured as the number of infected and isolated students reduced by the strategies.

Table 2. The parameters used in the social contacts network and SEIR model

\begin{tabular}{cll}
\hline Parameter & value & \\
\hline$p_{1}$ & 5 & Average number of one's contactors who are roommates \\
$p_{2}$ & 3.5 & Average number of one's contactors who are classmates but are living in other rooms \\
$p_{3}$ & 0.5 & Average number of one's contactors who are students belong to other classes \\
$\beta$ & 0.18 & Spreading rate \\
$1 / \gamma$ & 1.5 & Average infectious period \\
$1 / \varepsilon$ & 2 & Average latent period \\
$\lambda$ & 0.004 & The probability that a student is infected by outsiders in the first period \\
$\eta$ & 1 & The probability that edges between rooms are removed in the third period \\
$\theta$ & 0.8 & The probability that close contactors living in other rooms can be found during the outbreak \\
\hline
\end{tabular}

\section{Result}

\subsection{Social Contact Network and Stochastic Simulation}

The number of the newly infected in the building each day is shown in Fig. 3. In the first period, the infection was mainly introduced into the building from the outside and spread over more and more students. The transmission peaked in the second period and then reduced rapidly. If we take into account the 2-day latent period of the $\mathrm{H} 1 \mathrm{~N} 1$ virus, we conclude that the number of students exposed to infection decreased quickly after the administration isolated the building. Furthermore, the infection rate maintained at an extremely low level after the contacts among rooms were prohibited.

We simulated the H1N1 virus spreading process using the social contact network with a community structure. The related parameters are listed in Table 2. As shown in Fig. 1, the students first form small groups through roommate relationships, then form larger clusters through connecting rooms belonging to the same classes, and at last form the entire contact network for the building. The simulation results, averaged over 100 experimental runs, turn out to fit the overall outbreak pattern very well, as shown in Fig. 3. The coefficient of determination between the number of infected students on each day in the real outbreak and simulation results is 0.79. Fig. 4 illustrates the relationship between infection transmission and social networks based on a 
typical single simulation run. It shows that inter-class transmission rarely exists. Within each class, frequent internal transmissions in rooms exist and the inter-room transmissions lead to infection spreading over the class.

We further observe that the transmission mode of the H1N1 outbreak is aligned with the community structure of the social contact network. Infection spread quickly among densely connected groups of people. Fig. 5(a) shows the distribution of infected number in dormitory rooms. There were a total of 68 dormitory rooms in which students have been got infected. Among these rooms, 44(64.7\%) room with only one infected case, the other $24(35.3 \%)$ rooms possessed more than two infected cases despite the fact that the roommates of the infected students were quarantined promptly. The results indicate that rooms are relatively independent units where the infection is rapidly spreading.

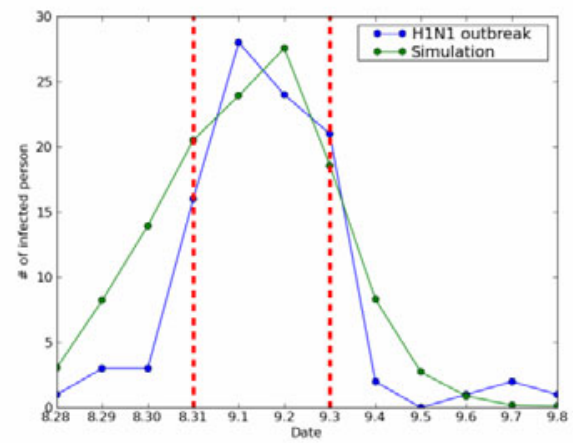

Fig. 3. The spreading process of the infection. Each point on the blue and green lines represents the number of the newly infected in the building each day of the situation of real outbreak and simulation, respectively. The outbreak is divided into three periods by the red dashed line.

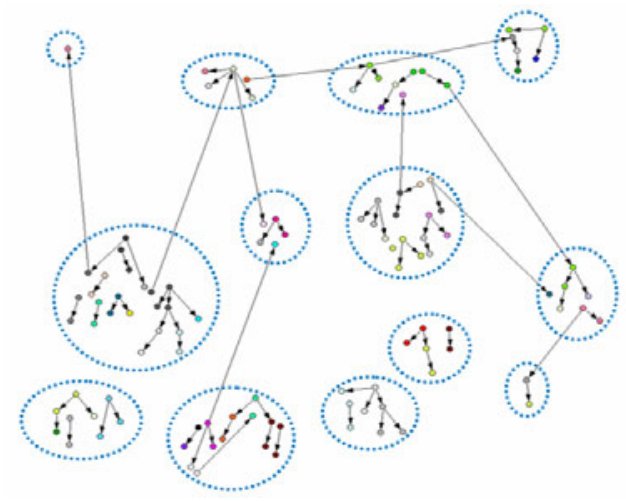

Fig. 4. The spreading process on the social contact network based on a typical single simulation run. Each point represents a student, and each edge represents a spreading path of the infection. The students are divided into various classes, denoted as circles. The students living in one room are marked with the same color. 
During the outbreak, the infection situation differed greatly across classes: the average infected number in each class is 7.5 whereas the standard deviation is 9.0. Note that the number of students across classes is quite uniform with the average being 136.7 and the standard deviation 18.2. As shown in Fig. 5(b), 12 classes housed less than $10 \%$ of infected students and two classes experienced larger outbreaks with $15(10 \%$ to $20 \%)$ cases and 32 cases $(30 \%-40 \%)$. This imbalance implies that an outbreak could be occurring in individual classes with limited inter-class interference.

In general, the simulation produced $\mathrm{H} 1 \mathrm{~N} 1$ transmission in small communities such as rooms and classes very similar to what was observed in the real outbreak. We measured the Pearson's correlation coefficient between the number of dormitory rooms (classes) which possessed different infection number in real outbreak and simulation results. We obtained a value of 0.99 (0.95 for the class, both with $p<0.005)$.

\subsection{Evaluation of Control Measures}

Isolating the whole building. When an outbreak occurred on a campus, isolation of buildings could help prevent epidemic spreading among buildings. As shown in Fig. 6 (a-b), comparing with isolating the whole building on the seventh day, taking the strategy on the first day can reduce the number of infected and isolated students in the entire outbreak by $85 \% \sim 92 \%$. The finding indicates that it is essential to strictly control visitations and identify the infected patients as soon as possible.

In practical terms, due to the high social and economic costs associated with building isolation, one needs to make careful decisions as to the timing of the isolation after H1N1 infection is reported in a school. In this model, the number of infected isolated students in the entire outbreak is 67 (300) fewer if the whole building is isolated on the first day than on the seventh day when $\lambda=0.001$. While, when $\lambda=0.007$, the building isolation taken on the first day would prevent 234 (988) students to get infected (isolated) compared to taking the strategy on the seventh day. In fact, we isolated the building on first day, only 26 infected and isolated 110 close contacts. The simulation results show that the more probable a student gets infected by an outsider, the more beneficial an earlier isolation.

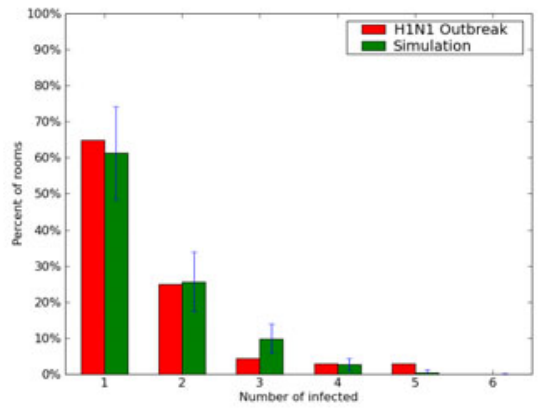

(a)

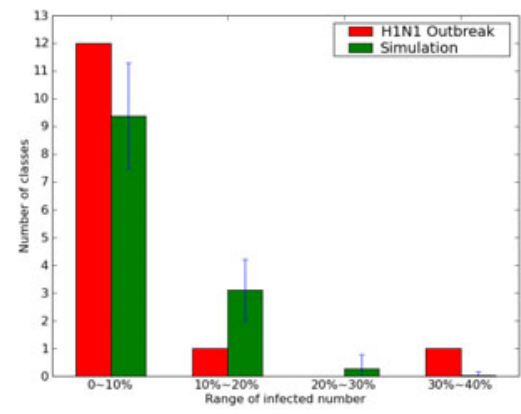

(b)

Fig. 5. (a) The percent of rooms versus the number of infection cases in one room. (b) The number of classes versus the ratio of infected number in one class to the sum infected in the building. 


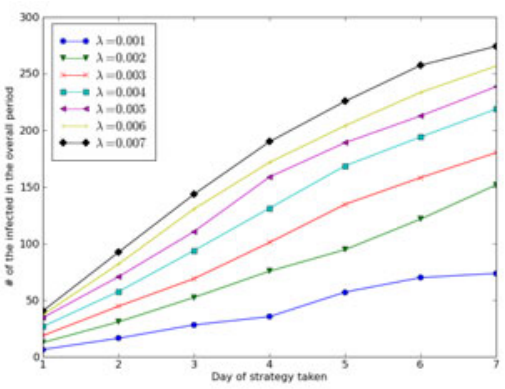

(a)

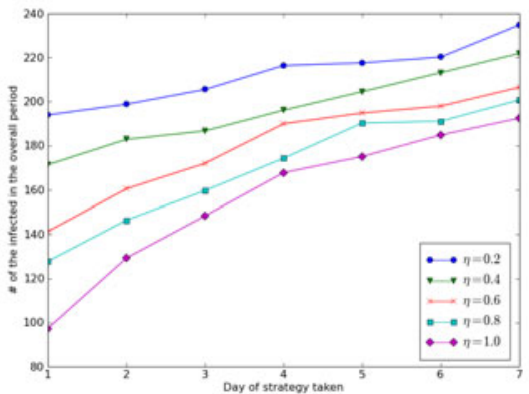

(c)

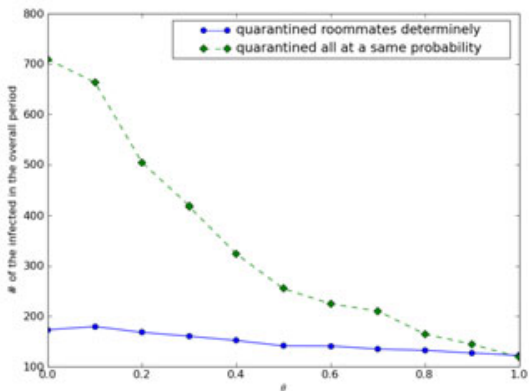

(e)

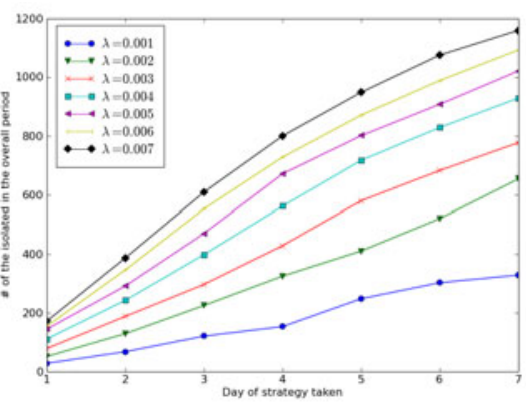

(b)

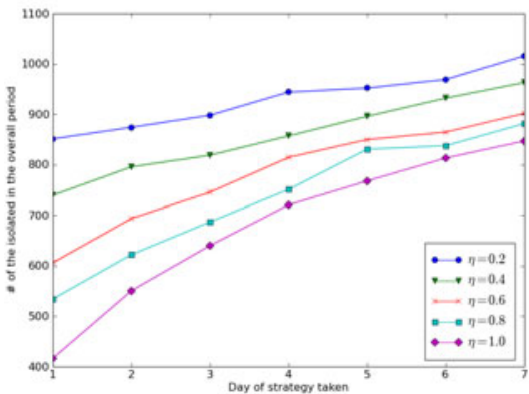

(d)

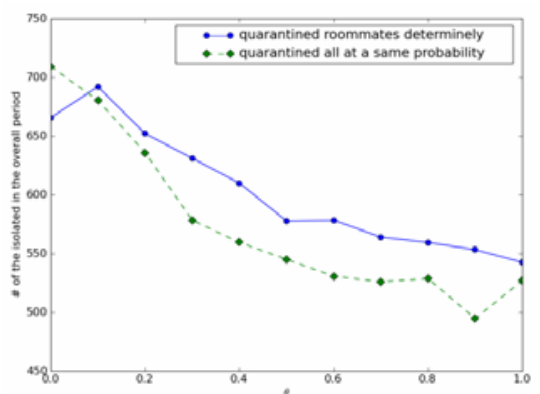

(f)

Fig. 6. The comparison of effectiveness of different control strategies under different setting. (a-b): The sum of (a) infected and (b) isolated versus the day that building is isolated, with $\lambda=$ $0.001,0.002,0.003,0.004,0.005,0.006,0.007$ from bottom to top, respectively. (c-d) The sum of (c) infected and (d) isolated versus the day that contact among rooms is cut after isolation of building, with $\eta=0.2,0.4,0.6,0.8,1.0$ from bottom to top, respectively. (e-f): The sum of (e) infected and (f) isolated versus the probabilities that close contacts are found. The solid line represents the roommates are determinedly quarantined and others are quarantined with specific probability denoted as $\theta$; the dash line represents a comparative method that all contacts are quarantined with the same probability denoted as $\theta$. 
Cutting off the contacts across rooms. We have found that frequent visitations between dormitory rooms can trigger clustered outbreaks within classes. Fig. 6 (c-d) shows the effect of reducing inter-room contacts on different dates after building isolation became effective on August 31, 2009. If we take the strategy on the first day, cutting off all contacts across rooms would prevent 97 (435) $(\eta=1.0$ vs $\eta=0.2)$ students to get infected (isolated) compared to the situation that $20 \%(\eta=0.2)$ contacts are cut off. While, the decreased ratio of infected/isolated students was $49.4 \% / 50 \%(42 / 169)$ $(\eta=1.0$ vs $\eta=0.2)$ when the strategy taken on the seventh day. The simulation results reveal that the impact of contact reduction measures on disease spread is minimum if these measures are taken after the outbreak is already progressing fast. From the policy perspective, the timing of the implementation needs to be carefully considered.

Quarantining the close contacts. Due to the existence of the latent period of the $\mathrm{H} 1 \mathrm{~N} 1$ virus, the close contacts of an infection case may be infected and contagious but without symptoms temporarily. As such, one possible outbreak containment strategy would be to identify and quarantine the close contacts exposed to the infection before symptoms show. We have found that the roommates have frequent contact with each other and need to be quarantined immediately when an infection case is found in a room. This observation leads us to experiment the control measure that isolate the infected person and his or her roommates during the simulation. Fig. 6 (e-f) shows the effectiveness of this measure. Without the previous knowledge of quarantining roommates of infected students, the incremental number of infected students is 536 when quarantining none of possible contacts; this drop to 114 when half of possible contacts are quarantined, regardless of the difficulties in accurately identifying possible contacts in time. Almost close contacts were found and quarantined, so the infected case (105) in this outbreak less than the simulated number(122) when $\theta=1.0$ (Fig. 6 (e)).Although the cost of quarantining all the roommates is high, the total number of students being isolated using this measure is almost the same as that of the alternative measure during the entire course of the outbreak(Fig.5 (f)) There were no difference $(P>0.05)$ on number of isolated whether quarantined the roommates and all close contacts or only probable contacts(542vs526) when $\theta=1.0$ (Fig. 6 (f)). As a result, we note that if the public health officials can quarantine the roommates of infected persons in a very short period of time, it is likely that the total number of infections can be significantly reduced.

\section{Discussion}

The dynamic of epidemic outbreak influenced by many factors including host immunity, virus virulence, human behavior, environmental change, social and economical situation, etc [10]. These factors influenced the virus transmission from human society, school to household, but social relationship as a special factor maybe influenced the transmission dynamic of epidemic outbreak in high density population [2-4, 6, 7]. In our research, we have investigated a real-world H1N1 outbreak occurred on a Chinese university campus. We have identified the community structure in students' social contact network and concluded that the community structure strongly affects the H1N1 spread and triggers clustered outbreaks. Based on our empirical findings, we have constructed a hierarchical social contact network model. Our computational 
experiments indicate that our model fits the real-world outbreak pattern very well and displays the clustering property of spreading through Monte-Carlo methods.

In this study, we investigated and identified a more comprehensive set of social factors influencing disease transmission of 2009 influenza A(H1N1) outbreak in density population special in school. The epidemic spread among susceptible people influenced by many social factors and identified by social network. For instance, the social contacts among students may be strongly influenced by their perception of the outbreak during the outbreak. Another significant result of the control measures used in this outbreak according the social relationship determined by models was effective and coordinated with models.

Using this social contact network model organized as a hierarchical network, we have conducted simulation studies to evaluate three public health response measures relevant to localized outbreaks: isolating the entire building, cutting off the contacts among rooms, and quarantining the close contacts of the infected. We have found that cutting off contacts among buildings and rooms can help contain the epidemic spread. As the H1N1 infection typically grows exponentially at first and tends to stabilize later [2], implementing the control measures earlier can be more beneficial.

The community structure of social contacts on campus leads to clustered outbreaks. On practical terms, we recommend that the roommates of the infected students be isolated as early as possible. The quickly infection spreading in the school requires emergency interventions, and the public measures implemented by the school administrator and our simulation, according to the community structure of social contacts, performs efficiently to respond to H1N1 outbreak in a localized area in time. On the contrary, prevaccination would be not practicable because vaccine cannot take effect instantly.

A limitation of our study is the lack of a quantitative analysis of cost-effectiveness of various measures. Real costs of a response measure are difficult to estimate, which include both social costs and economic costs caused by reducing contacts, providing isolation spaces, investigating contacts of the infected, handing out mask, taking protective measures and so on. The economic and social benefit of containing an outbreak is also hard to quantify, as it will be dependent on the virulence and clinical severity of illness, and the productivity and economic loss due to closed schools and so on. Nonetheless, we believe that our study provides a starting point to assist public health response decision-making during epidemic outbreaks in localized areas.

Acknowledgments. This work was supported in part by the Major-projects of Science and Technology Research (Grant No. 2009ZX10004-315, 2008ZX10004-008, 2008ZX10005-013), the Chinese Academy of Sciences (Grants No. 2F07C01, 2F08N03), National Natural Science Foundation of China (Grant No. 90924302, 60621001, 60921061, 40901219, 71050001, 91024030), and the US National Science Foundation (Grant No. IIS-0839990).

\section{References}

1. Kermack, W.O., McKendrick, A.G.: Contributions to the mathematical theory of epidemics. Proceedings of the Royal Society of London 115, 700-721 (1927)

2. Barthélemy, M., Barrat, A., Pastor-Satorras, R., Vespignani, A.: Dynamical patterns of epidemic outbreaks in complex heterogeneous networks. Journal of Theoretical Biology 235, 275-288 (2005) 
3. Kiss, I.Z., Green, D.M., Kao, R.R.: The effect of network mixing patterns on epidemic dynamics and the efficacy of disease contact tracing. Journal of The Royal Society Interface 5, 791-799 (2008)

4. Moore, C., Newman, M.E.J.: Epidemics and percolation in small-world networks. Physical Review E 61, 5678 (2000)

5. Newman, M.: Modularity and community structure in networks. Proceedings of the National Academy of Sciences 103, 8577 (2006)

6. Yang, Y., Sugimoto, J., Halloran, M., Basta, N., Chao, D., Matrajt, L., Potter, G., Kenah, E., Longini Jr., I.: The transmissibility and control of pandemic influenza A (H1N1) virus. Science 1177373v1177371 (2009)

7. Cauchemez, S., Bhattarai, A., Marchbanks, T.L., Fagan, R.P., Ostroff, S., Ferguson, N.M., Swerdlow, D.: Role of social networks in shaping disease transmission during a community outbreak of 2009 H1N1 pandemic influenza. Proceedings of the National Academy of Sciences (2009)

8. Lipsitch, M., Cohen, T., Cooper, B., Robins, J.M., Ma, S., James, L., Gopalakrishna, G., Chew, S.K., Tan, C.C., Samore, M.H., Fisman, D., Murray, M.: Transmission Dynamics and Control of Severe Acute Respiratory Syndrome. Science 300, 1966-1970 (2003)

9. Ou, J., Dun, Z., Li, Q., Qin, A., Zeng, G.: Efficiency of the quarantine system during the epidemic of severe acute respiratory syndrome in Beijing. Zhonghua liu xing bing xue za zhi= Zhonghua liuxingbingxue zazhi 24, 1093 (2003)

10. Grassly, N.C., Fraser, C.: Mathematical models of infectious disease transmission. Nat. Rev. Micro. 6, 477-487 (2008)

11. Han, K., Zhu, X., He, F., Liu, L., Zhang, L., Ma, H., Tang, X., Huang, T., Zeng, G., Zhu, B.: Lack of airborne transmission during outbreak of pandemic (H1N1) 2009 among Tour Group Members. Emerging Infectious Disease 10, 1578-1581 (2009)

12. Shen, Z., Ning, F., Zhou, W., He, X., Lin, C., Chin, D., Zhu, Z., Schuchat, A.: Superspreading SARS events, Beijing. Emerging Infectious Diseases 10, 256-260 (2004)

13. ZhiDong, C., DaJun, Z., QuanYi, W., XiaoLong, Z., FeiYue, W.: An epidemiological analysis of the Beijing 2008 Hand-Foot-Mouth epidemic. Chinese Science Bulletin 55, 1142-1149 (2010)

14. Cao, Z.D., Zeng, D.J., Zheng, X.L., Wang, Q.Y., Wang, F.Y., Wang, J.F., Wang, X.L.: Spatio-temporal evolution of Beijing 2003 SARS epidemic. Science China Earth Sciences, $1-12$ (2010)

15. Bondy, S., Russell, M., Lafleche, J., Rea, E.: Quantifying the impact of community quarantine on SARS transmission in Ontario: estimation of secondary case count difference and number needed to quarantine. BMC Public Health 9, 488 (2009)

16. Yasuda, H., Suzuki, K.: Measures against transmission of pandemic H1N1 influenza in Japan in 2009: simulation model. European Communicable Disease Bulletin 14 (2009)

17. Hsieh, Y., King, C., Chen, C., Ho, M., Hsu, S., Wu, Y.: Impact of quarantine on the 2003 SARS outbreak: A retrospective modeling study. Journal of Theoretical Biology 244, 729736 (2007)

18. Dan, Y., Tambyah, P., Sim, J., Lim, J., Hsu, L., Chow, W., Fisher, D., Wong, Y., Ho, K.: Cost-effectiveness Analysis of Hospital Infection Control Response to an Epidemic Respiratory Virus Threat. Emerging Infectious Disease 15, 1909-1916 (2009)

19. Dasgupta, K., Menzies, D.: Cost-effectiveness of tuberculosis control strategies among immigrants and refugees. European Respiratory Journal 25, 1107 (2005)

20. Newman, M., Barabasi, A., Watts, D.: The structure and dynamics of networks. Princeton Univ. Pr., Princeton (2006) 
21. Pastor-Satorras, R., Vespignani, A.: Epidemic Spreading in Scale-Free Networks. Physical Review Letters 86, 3200 (2001)

22. Zheng, X.L., Zeng, D., Li, H.Q., Wang, F.Y.: Analyzing open-source software systems as complex networks. Physica A 387, 6190-6200 (2008)

23. Yin, H., Rong, Z., Yan, G.: Development of friendship network among young scientists in an international Summer School. Physica A: Statistical Mechanics and its Applications 388, 3636-3642 (2009)

24. Girvan, M., Newman, M.E.J.: Community structure in social and biological networks. Proceedings of the National Academy of Sciences of the United States of America 99, 78217826 (2002)

25. Danon, L., Arenas, A., Díaz-Guilera, A.: Impact of community structure on information transfer. Physical Review E 77, 036103 (2008)

26. Wu, J.-j., Gao, Z.-y., Sun, H.-j.: Cascade and breakdown in scale-free networks with community structure. Physical Review E 74, 066111 (2006)

27. Hethcote, H.: The mathematics of infectious diseases. SIAM Review 42, 599-653 (2000)

28. Tuite, A.R., Greer, A.L., Whelan, M., Winter, A.L., Lee, B., Yan, P., Wu, J., Moghadas, S., Buckeridge, D., Pourbohloul, B.: Estimated epidemiologic parameters and morbidity associated with pandemic H1N1 influenza. Canadian Medical Association Journal 182, $131(2010)$

29. Canini, L., Carrat, F.: Population Modeling of Influenza A/H1N1 Virus Kinetics and Symptom Dynamics. J. Virol. 85, 2764-2770 (2011) 